\title{
Usefulness of a 1,064 nm Microlens Array-type, Picosecond- dominant Laser for Pigmented Scars with Improvement of Vancouver Scar Scale
}

\author{
Kwang Hyeon Ahn \\ Eun Soo Park \\ Seung Min Nam
}

Department of Plastic \& Reconstructive Surgery, Soonchunhyang University Bucheon Hospital, Bucheon, Korea
Received June 26, 2019

Accepted June 26, 2019

Correspondence
Eun Soo Park
Department of Plastic and Reconstructive
Surgery, Soonchunhyang University Bucheon
Hospital, 1174 Jomaru-ro, Wonmi-gu, Bucheon
14584, Korea
Tel.: +82-32-621-5319
Fax: +82-32-621-5016
E-mail: peunsooldschmc.ac.kr
(c) Korean Society for Laser Medicine and Surgery
@c This is an open access article distributed under the
terms of the Creative Commons Attribution Non-
Commercial License (http://creativecommons.org/
licenses/by-nc/4.0) which permits unrestricted non-
commercial use, distribution, and reproduction in any
medium, provided the original work is properly cited.

\begin{abstract}
Background and Objectives
The picosecond $755 \mathrm{~nm}$ alexandrite laser was first approved by the US FDA in 2012. A previous study described the use of a $1,064 \mathrm{~nm}$ picosecond laser with a micro-lens array (MLA) in peri-areolar scarring from breast reconstruction surgery and reported significant improvement in the texture and aesthetic appearance of the scar without other wound complications. The purpose of this study was to evaluate the improvement of overall scarring, not just pigmentation, in the picosecond laser treatment of patients with pigmentations.
\end{abstract}

\section{Materials and Methods}

Sixteen patients who underwent 1,064 nm picosecond laser treatment from June 2016 to December 2018 were enrolled in this study. Patients received two to six sessions of picosecond laser treatment at intervals of 4 weeks. Before and after the laser treatment, the patients evaluated their own satisfaction score and a physician evaluated the Vancouver Scar Scale. To evaluate the satisfaction score and complication rate, a retrospective chart review was done.

\section{Results}

Seven were female and nine were male. The mean of the patients' satisfaction score before the treatment was 1.44 (interquartile range [IQR], 1-2) and 3.00 (IQR 2.25-3.75) six months after treatment. The mean of the Vancouver Scar Scale before the treatment was 9.69 (IQR 8-11), and 6.25 (IQR 5-7.75) six months after treatment. All the results were statistically significant $(p<0.01)$.

\section{Conclusion}

This study provides evidence that the use of a 1,064 nm picosecond laser treatment for pigmented scars can be effective in improving the pigmentation and overall scar status, including vascularity, height, and pliability, with the results of a decrease in the VSS scores between treatments.

\section{Key words}

Laser therapy; Pigmented scars; Vancouver Scar Scale; Picosecond laser 


\section{INTRODUCTION}

The picosecond $755 \mathrm{~nm}$ alexandrite laser was first approved by the US FDA in 2012 after its success for removal of tattoos requiring less treatment than the traditional nanosecond quality switched (Q-switched) lasers.' This picosecond laser with an ultra-short pulse duration of 550 to 750 picosecond is currently approved by US FDA for not only the removal of tattoos, but also the treatment of wrinkles and acne scarring. With a specific wavelength, the energy of laser is absorbed by a target melanocytic lesion, resulting destroying the pigmentation without damaging the normal tissue. The rapid absorption of picosecond laser light destroy the melanin, leaving the treated skin uniform in color and texture. ${ }^{2}$

Prior studies focused on using picosecond laser in facial wrinkles and acne scarring. There was only limited case report about using picosecond laser in other types of scarring, not only in acne scarring. Our previous study described the use of 1,064 nm picosecond laser with microlens array (MLA) in peri-areolar scarring from breast reconstruction surgery showed significant improvement in the texture and aesthetic appearance of the scar without other wound complication. ${ }^{3}$ The purpose of this study was to evaluate the improvement of overall scarring, not only pigmentation, in the treatment of picosecond laser in patients with pigmentations.

\section{MATERIALS AND METHODS}

\section{Patients}

Of Patients who underwent pigmentation wound treatment using picosecond laser between June 2016 and December 2018, 16 patients who could be observed throughout a follow-up of longer than 6 months were enrolled in this study. Patients with systemic or local infection of wound, psychiatric diseases or history of surgical procedure like scar revision were excluded.

Patients' medical charts and operative records were reviewed retrospectively to evaluate outcomes and complications before and after the picosecond laser treatment. This study conformed to the Declaration of Helsinki. Written consents were obtained from each patient for both the laser treatment and the publication of photographs of the results.

\section{Preparation before laser treatment}

In all patients applied a 5\% lidocaine topical anesthetic ointment (Emla ${ }^{\circledR}$ AstraZeneca AB, Karlskoga, Sweden) to the target area before the picosecond laser treatment.
This topical anesthetic ointment was washed off with mild soap and water immediately before the procedure.

\section{Picosecond laser treatments}

A total of 16 patients were treated with PICO laser (PICOCARE $^{\circledR}$; WONTECH, Daejeon, Korea), at a wavelength of $1,064 \mathrm{~nm}$ picosecond, a spot size of $3 \mathrm{~mm}$ to $10 \mathrm{~mm}$, fluence of $3.8 \mathrm{~J} / \mathrm{cm}^{2}$ to $5.1 \mathrm{~J} / \mathrm{cm}^{2}$, pulse width of 1,064 picosecond, and frequency of 8 to $10 \mathrm{~Hz}$, in combination with an MLA. Each patient underwent 2 to 6 sessions of laser. Photographic findings were taken before and after the each round of laser treatment.

\section{Care after laser treatment}

A topical antibiotic ointment was applied to the target area after each laser treatment session. We educated all patients to avoid direct sunlight and recommended to apply a sunscreen agent between the laser treatment sessions for minimizing post-inflammatory hyperpigmentation. We educated the patients to visit hospital immediately when they had any adverse effect.

\section{Evaluation of outcomes}

At the end of the laser treatment, the patients were asked to rate their satisfaction with the improvements in the aesthetic appearance and texture of the scars using a 4-point scale (0, not satisfied; 1, dissatisfied; 2, satisfied; and 3, extremely satisfied). In addition, 1 plastic surgeon evaluated the improvement of the patients' scars using the Vancouver Scar Scale (Fig. 1) before the initial treatment and 6 months after the final treatment.

\section{Statistical analysis}

Statistical analyses were performed using SPSS version

$\begin{array}{ll}\text { Vascularity } & \text { Normal } \\ & \text { Pink } \\ & \text { Red } \\ & \text { Purple } \\ \text { Pigmentation } & \text { Normal } \\ & \text { Hypopigmentation } \\ & \text { Hyperpigmentation } \\ \text { Pliability } & \text { Normal } \\ & \text { Supple } \\ & \text { Yielding } \\ & \text { Firm } \\ & \text { Ropes } \\ & \text { Contracture } \\ \text { Height } & \text { Flat } \\ & <2 \mathrm{~mm} \\ & 2-5 \mathrm{~mm} \\ & >5 \mathrm{~mm} \\ & \text { Total score }\end{array}$

Fig. 1. Vancouver Scar Scale. 
24.0 (IBM Corp., Armonk, NY, USA). The Wilcoxon signedrank test was used to compare the patients' satisfaction scores and the Vancouver Scar Scale before and after the picosecond laser treatment. Statistical significance was considered to be $p<0.05$.

\section{RESULTS}

Of the 16 patients treated with picosecond laser, 7 were female and 9 were male. The mean age of the patients was 39.1 years (range, 17-67 years) and the mean followup period was 9.2 months (range, 6-16 months) (Table 1).

The mean of patients' satisfaction score was 1.44 (interquartile range [IQR], 1-2) before the treatment, and 3 (IQR 2.25-3.75) 6 months after the treatment (Fig. 2 and Table 2). This improvement of patients' subjective satisfaction score was statistically significant $(p<0.01)$.

Table 1. Patients' characteristics

\begin{tabular}{lc}
\hline Characteristics & Value \\
\hline Sex & \\
Male & $9(56.3 \%)$ \\
Female & $7(43.7)$ \\
Age (year), mean (range) & $39.1(17-67)$ \\
\hline
\end{tabular}

The mean of the Vancouver Scar Scale was 9.69 (IQR 8-11) before the treatment, and 6.25 (IQR 5-7.75) 6 months after the treatment (Fig. 3 and Table 2). This decrease of the Vancouver Scar Scale was statistically significant $(p<0.01)$.

\section{DISCUSSION}

There is high demand of cosmetic laser treatment in patients with various skin problem. $\mathrm{CO}_{2}$ lasers were most commonly used laser device in treating surgical scars. Hong et al. used early fractional $\mathrm{CO}_{2}$ lasers (Line-Xel) 4 weeks after surgery and trauma and proved it effective in minimizing scar formation. ${ }^{10}$ Cho et al. used $\mathrm{CO}_{2}$ lasers (Line-Xel) in treating local flap surgical scar and found it effective in improving the Vancouver Scar Scale. ${ }^{11}$

Since 2012 when the FDA approved use of $755-n m$ picosecond alexandrite laser in removing tattoos, the picosecond laser has been used to treat various pigmented scars and showed their effectiveness in pigmented scars. Torbeck et al. ${ }^{4}$ conducted systemic review of 41 articles about the effectiveness of picosecond laser in pigmented scars, removing tattoos and acne scarring. In most studies, the result was effective and safe.

As the effectiveness and safeness of picosecond laser

Table 2. Scar evaluation tools

\begin{tabular}{cccc}
\hline Time & Pre-treatment (median) & Post-treatment (median) & $p$-value \\
\hline Patients' satisfaction & 1.44 (IQR:1-2) & 3 (IOR:2.25-3.75) & $<0.01$ \\
Vancouver Scar Scale & 9.69 (IQR:8-11) & 6.25 (IOR:5-7.75) & $<0.01$ \\
\hline
\end{tabular}

IQR, interquartile range.

P-value by Wilcoxon signed-rank test.

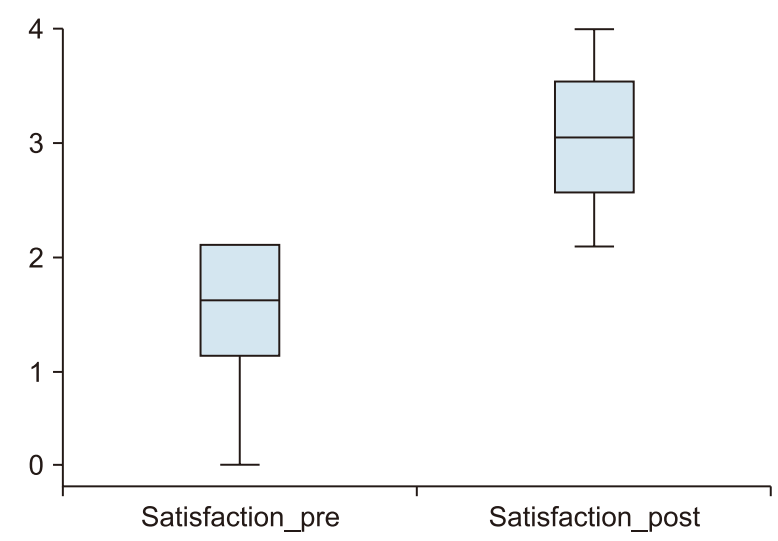

Fig. 2. The mean of patients' satisfaction score was 1.44 (interquartile range [IQR], 1-2) before the treatment, and 3 (IQR 2.25-3.75) 6 months after the treatment.

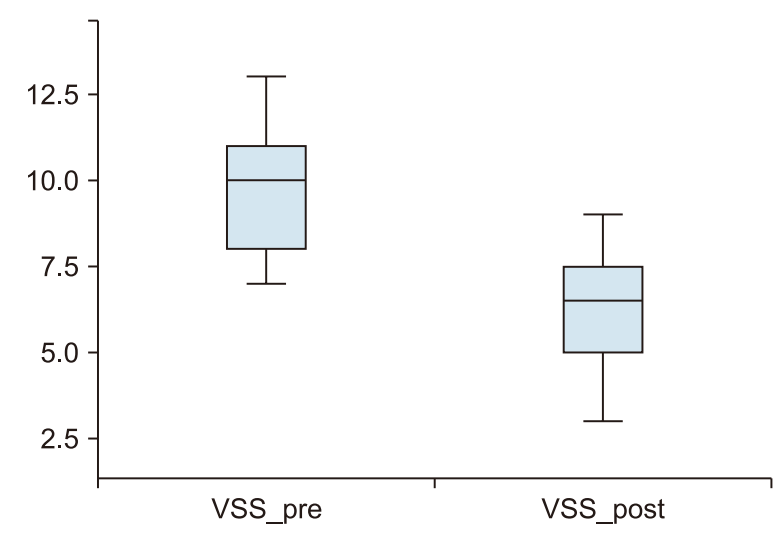

Fig. 3. The mean of the Vancouver Scar Scale was 9.69 (IQR 8-11) before the treatment, and 6.25 (IQR 5-7.75) 6 months after the treatment. 
was approved, many dermatologist and plastic surgeons have paid attention to the wide range of use of picosecond laser in various skin problems, not only tattoos. Brauer et al. ${ }^{5}$ tried to investigate the safety and efficacy of a 755-nm picosecond laser for the treatment of facial acne scarring. Their results showed reliable improvement in appearance and texture at 3 months after the laser treatment. The histologic findings suggest the improvement in scarring from remodeling of collagens in scar tissues.

FDA approved use of picosecond laser treatment with ultra-short duration for the treatment of wrinkles and acne scarring since 2014. The mechanism of picosecond laser in improving scarring is based on laser-induced optical breakdown (LIOB). Picosecond laser with a Micro Lens Array (MLA) can concentrate energy in target spots to produce more high energy. Melanocytes in the epidermal zones absorb the energy and free electrons are generated. The laser light is absorbed by this free electrons, resulting in the formation of second free electrons. This cascade of generating free electrons finally create an ionized plasma absorbing remaining laser radiation. In the last step of LIOB, a vacuole is created in the epidermis when the plasma has absorbed sufficient energy. This cavitation phenomenon can release the contracted collagen tissues in scarring, leading the improvement of scars like sub-incision procedure. Also, this cavitation in epidermal layer results in a rejuvenation effect, producing new dermal collagen, elastic tissue and mucin., 3

In our study, the patients received 2 to 6 times of picosecond laser treatment at 4 weeks interval. We found a statistically significant improvement of patients' satisfaction score. The patients' satisfaction score is a subjective way of evaluating the status of scars. However it is important criteria, as they conclude the effectiveness of laser
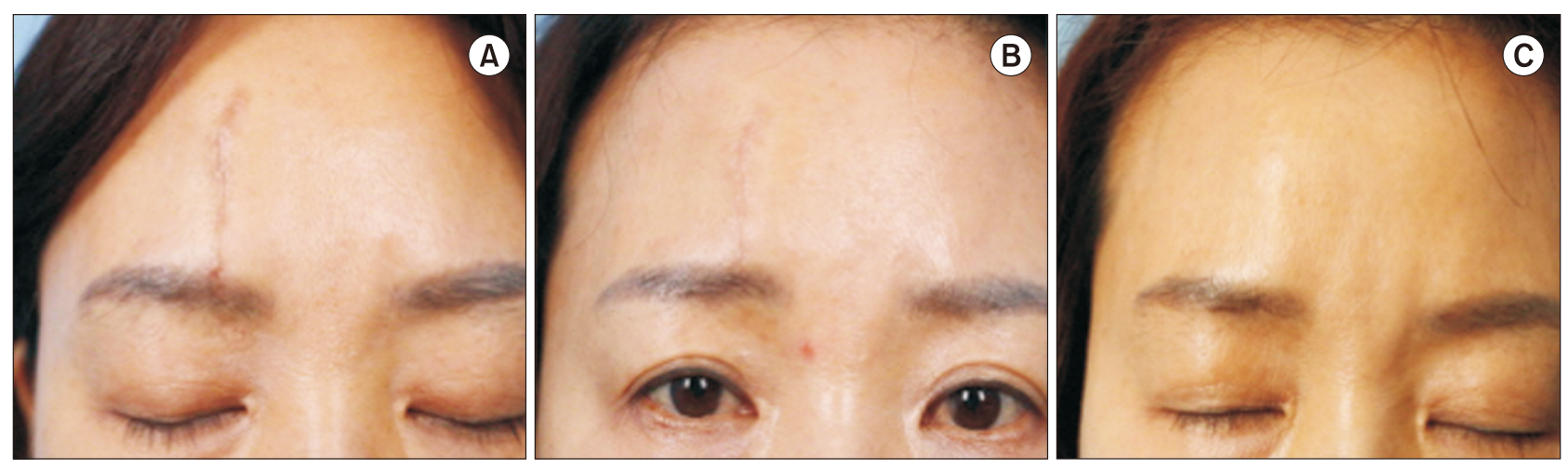

Fig. 4. A 53-year-old female patient with forehead scar with pigmentation (A). Before the treatment, the VSS was 11. In first treatment session, the parameter was spot size of $10 \mathrm{~mm}$, fluence of $3.1 \mathrm{~J} / \mathrm{cm}^{2}$ and frequency of $8 \mathrm{~Hz}$ with 783 shots. In the second session, the parameter was spot size of $3 \mathrm{~mm}$, fluence of $3.8 \mathrm{~J} / \mathrm{cm}^{2}$ and frequency of $8 \mathrm{~Hz}$ with 226 shots. After final treatment sessions (B), and 4 months after the final treatment (C). After the final treatment, the VSS was 4. Dynamic improvement of the pigmentation and height could be observed.
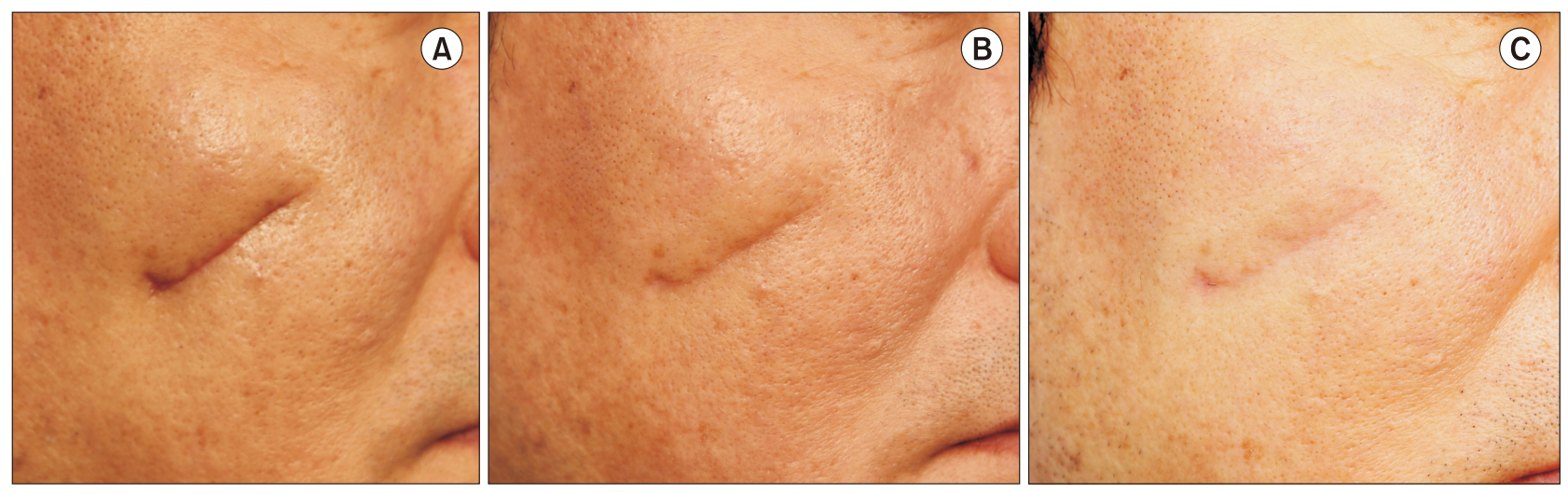

Fig. 5. A 46-year-old male patient with cheek scar with pigmentation (A). Before the treatment, the VSS was 13. In first treatment session, the parameter was spot size of $3 \mathrm{~mm}$, fluence of $5.1 \mathrm{~J} / \mathrm{cm}^{2}$ and frequency of $9 \mathrm{~Hz}$ with 435 shots. In the second and third session, the parameter was same as the first session and the total shots were 287 and 1060 shots for each sessions. After final treatment sessions (B), and 6 months after the final treatment (C). After the final treatment, the VSS was 5. 
treatment according to their own satisfaction.

Our study aimed to determine the efficacy of picosecond laser treatment in the improvement of scars, not only the pigmentation. So we tried to evaluate the overall scars with the Vancouver Scar scales (VSS). The Vancouver Scar scales were first was first introduced in 1990 and has been validated and extensively described in the literature. ${ }^{7.8}$ This scoring system focuses on 4 parameters; scar height and thickness, pliability, vascularity, and pigmentation to generate score ranging from 0 to 13 points. ${ }^{9}$ Physicians evaluate the scars using the VSS. When the more scars are similar with normal tissue, the lower VSS is. In our study, the mean of VSS before the treatment was 9.69 after two sessions of picosecond laser treatment for the pigmentation wound, the mean of VSS decreased to 6.25 and it was statistically significant. Also, when it comes to only the pigmentation parameter, the mean score was decreased from 2 to 0.86 , which was statistically significant (Fig. 4, 5).

Our study had limitations. First, the histologic evaluation was not conducted in our study. Therefore, for more objective studies, histologic evaluation by tissue biopsy would be included in next study. Second, the mean follow up period was 6 months. So the long term follow up results were limited, which would be important in evaluating the final results of scarring improvement. Despite these limitations, our study showed that the improvement of Vancouver Scar scale sores in patients treated with picosecond laser, and proved it statistically significant.

\section{CONCLUSION}

We provide evidence that the use of 1,064 $\mathrm{nm}$ picosecond laser treatment for the pigmented scars can be effective in not only the pigmentation improvement but also the overall scar status, including vascularity, height, and pliability, with the results of decrease of VSS scores between the treatments. The length of follow-up was only 6 months, so long-term period evaluation of safety and efficacy would be needed for next studies.

\section{ACKNOWLEDGEMENTS}

This work was supported by the Soonchunhyang Uni- versity Research Fund.

\section{CONFLICT OF INTEREST}

The authors report no conflicts of interest.

\section{REFERENCES}

1. Jakus J, Kailas A. Picosecond lasers: a new and emerging therapy for skin of color, minocycline-induced pigmentation, and tattoo removal. J Clin Aesthet Dermatol 2017;10:14-5.

2. Khetarpal S, Desai S, Kruter L, Prather H, Petrell K, Depina J, et al. Picosecond laser with specialized optic for facial rejuvenation using a compressed treatment interval. Lasers Surg Med 2016;48:723-6.

3. Choi Y, Park ES. Subsurface fractional ablative resurfacing of a periareolar scar using 1,064-nm picosecond laser with microlens array. Arch Aesthetic Plast Surg 2018;24:36-8.

4. Torbeck RL, Schilling L, Khorasani H, Dover JS, Arndt KA, Saedi N. Evolution of the picosecond laser: a review of literature. Dermatol Surg 2019;45:183-94.

5. Brauer JA, Kazlouskaya V, Alabdulrazzaq H, Bae YS, Bernstein LJ, Anolik R, et al. Use of a picosecond pulse duration laser with specialized optic for treatment of facial acne scarring. JAMA Dermatol 2015;151:278-84.

6. Tanghetti EA. The histology of skin treated with a picosecond alexandrite laser and a fractional lens array. Lasers Surg Med 2016;48:646-52.

7. Sullivan T, Smith J, Kermode J, Mclver E, Courtemanche DJ. Rating the burn scar. J Burn Care Rehabil 1990;11:256-60.

8. Thompson CM, Sood RF, Honari S, Carrougher GJ, Gibran NS. What score on the Vancouver Scar Scale constitutes a hypertrophic scar? Results from a survey of North American burncare providers. Burns 2015;41:1442-8.

9. Nguyen TA, Feldstein SI, Shumaker PR, Krakowski AC. A review of scar assessment scales. Semin Cutan Med Surg 2015;34:28-36.

10. Hong SC, Park ES, Kim YB, Nam SM. Effects of minimizing scar formation by early fractional $\mathrm{CO} 2$ laser resurfacing. Arch Aesthetic Plast Surg 2014;20:109-13.

11. Cho SE, Nam SM, Kim YB, Park ES. Local flap surgical scar management caused by skin cancer using fractional CO2 laser treatment. Arch Aesthetic Plast Surg 2015;21:18-22. 